\title{
Hardware Synthesize and Performance Analysis of Intelligent Transportation Using Canny Edge Detection Algorithm
}

\author{
Aisha Baloch \\ Institute of Information Communication and Technologies, Mehran University of Engineering and Technology, \\ Jamshoro, Pakistan \\ E-mail: aisha.13muet@gmail.com
}

\section{Tayab D Memon}

Department of Electronic Engineering, Mehran University of Engineering and Technology, Jamshoro, Pakistan. School of Information Technology and Engineering, Melbourne Institute of Technology, Melbourne, Australia Email: tmemon@mit.edu.au

\section{Farida Memon}

Department of Electronic Engineering, Mehran University of Engineering and Technology, Jamshoro, Pakistan. Email: farida.memon@faculty.muet.edu.pk

\section{Bharat Lal}

Department of Electronic Engineering, Mehran University of Engineering and Technology, Jamshoro, Pakistan. Email: bharat.lal@faculty.muet.edu.pk

\section{Ved Viyas}

Institute of Information Communication and Technologies, Mehran University of Engineering and Technology, Jamshoro, Pakistan

E-mail: vedmeghwar@gmail.com

\section{Tony Jan}

School of Information Technology and Engineering, Melbourne Institute of Technology, Melbourne, Australia

Received: 10 April 2021; Accepted: 16 June 2021; Published: 08 August 2021

\begin{abstract}
The World is moving toward Smart traffic management and monitoring technologies. Vehicle detection and classification are the two important features of intelligent transportation system. Several algorithms for detection of vehicles such as Sobel, Prewitt, and Robert etc. but due to their less accuracy and sensitivity to noise they could not detect vehicles clearly. In this paper, a simple and rapid prototyping approach for vehicle detection and classification using MATLAB Xilinx system generator and Zedboard is presented. The Simulink model of vehicle detection and classification is designed using a complex canny edge detection algorithm for vehicle detection. The canny edge detection algorithm offers $91 \%$ accuracy as compared to its counterpart Sobel and Perwitt algorithms that offer 79.4\% and $76.1 \%$ accuracy. The feature vector approach is used for vehicle classification. The proposed model is simulated and validated in MATLAB. The Canny edge detection and feature vector algorithms for vehicle detection and classification are synthesized through the Xilinx system generator in Zedboard. The proposed design is validated with the existing works. The implementation results reveal that the proposed system for vehicle detection and classification takes only $8 \mathrm{~ns}$ of execution time with a $128 \mathrm{MHz}$ clock, which is the lowest and optimum calculation period for the smart city.
\end{abstract}

Index Terms: Intelligent Transportation, vehicle detection and classification, Xilinx system generator, Zedboard FPGA board, Xilinx Platform, DVI connector. 


\section{Introduction}

With the advent of the recent biggest technologies, the world is moving toward smart cities traffic management and monitoring i.e., intelligent transportation. Intelligent transportation requires detecting and identifying vehicles with significant features. Therefore, vehicle detection and classification are two important challenging tasks for traffic monitoring and real time collision management. Vehicle detection provides information about the localization of the vehicles. Each type of vehicles has their own unique features, and vehicles can be classified as either car, bus, or motorcycle based on those features [1]. The real-time image processing is rather resource intensive, and there is a great demand to consider an intelligent methodology to enable accurate and high-performance real-time image detection and classifications using reduced computing resources with low power - to fulfil the requirements of future applications in smart environments. The image processing application generally includes detection of the edges of a vehicle followed by vehicle type classifications. During the detection of vehicles, a well-known edge detection algorithm such as Canny is considered amongst other available options.

Edge detection is the vital part in image processing to detect an object of interest. The edge detection is to extract desired information and filter out unwanted information in object detection and further classifications.

The edge detection recognizes and locates sharp discontinuities and variations in an image. These discounters are the changes in the intensity of a pixel that may define the boundaries of an object in an image or the boundary between different regions of an image. Many computer vision applications, such as edge-based obstacle detection, edge-based vehicle recognition, or edge-based target recognition begin with these object bounders. The well-established edge detection methods include Sobel, Prewitt, and Canny as briefly summarized below.

Edge detection is a method to detect the presence of an object's image. Edge detection is used in fields such as image processing, computer vision, and machine vision for image segmentation and data extraction. The main goal of edge detection is to locate and identify sharp discontinuities from an image. These discontinuities are due to abrupt changes in pixel intensity which characterizes boundaries of objects in a scene as well as give boundaries between different regions in the image. Such boundaries are used to identify objects. These object boundaries are the first step in many computer vision algorithms like edge-based obstacle detection, edge-based Vehicle recognition, and edge-based target recognition Quality of edge detection plays a very important role in the focused area selection, image segmentation and object recognition. [8].For edge detection, several methods have been proposed [2.3] including, Sobel, Prewitt, Canny. The relevant literature review is presented in the following paragraphs that is followed by the brief on these edge detection algorithms.

In [4] Hardware Co-simulation of Underwater Moving Object Detection using the Xilinx System Generator system is presented. The only feature of the proposed system is the detection of the vehicle at the cost of a very high chip area and high computational time. In [5] FPGA-based Image Feature Extraction Using Xilinx System Generator with Sobel edge detection algorithm is implemented over Spartan-3E FPGA platform for edge detection. It is proposed that Sobel has a better algorithm in comparison to Prewitt with less noise in images. In [6] a Real-time hardware-software embedded vision system for ITS smart camera is implemented in Zynq SoC Using Vertex 4 platform, performing vehicle detection, counting, and classification. It is observed that the system could not classify vehicles. In [7], the work is proposed on image and video processing applications using Xilinx System Generator. The proposed design is only for vehicle detection with less accuracy. In [8] an evaluation of the Canny and Sobel Edge detection technique using Xilinx System Generator is presented. It is reported that the canny edge detection method gives a sharp edge image compared to the Sobel method.

In [9] an intelligent transportation system is proposed for the detection and classification of vehicles for traffic video analytics using the SVM algorithm. This work has no hardware synthesis to measure the area-performance and power utilization. Thus the Objective of the research is to design a prototype for intelligent transportation system that can detect and identify the vehicle using a canny edge algorithm for edge detection and feature vector approach for classification of vehicle type and to Synthesize and Analyse area-performance in the constraint environment using FPGA zedboard 7020.

The rest of the paper proceeds as follows. In section II, vehicle detection (i.e., edge detection algorithms) and vehicle classification approaches are described. In section III, the proposed system design in the MATLAB Xilinx system generator is presented that is followed by the system implementation in section IV. In section V, the system simulation and results are presented that are followed by the conclusion in section $\mathrm{V}$.

\section{Vehicle Detection and Classification Approaches}

In the first part, we present the three edge detection algorithms i.e., Sobel, Prewitt, and Canny Edge. In the second part, the vehicle classification approaches are presented.

\section{A. Edge Detection Algorithms}

Sobel Edge Detection is a technique based on two measuring $3 \times 3$ Convolution kernels. one kernel detects the 
horizontal edges and the other kernel recognizes the vertical edges. Each kernel has the effect of calculating the gradient in both a horizontal and a vertical direction. The image is read initially after the start i.e. the pixel values are read. The kernels are applied separately to the input image to produce gradient components measurements separately The image is then convolved with the filter. After that horizontal and vertical kernels of the operator are convolved with the original image [10]. the gradient calculated by Sobel operator is given below:

$$
\mathrm{G}=\sqrt{G x^{2}+G y^{2}}
$$

Information:

G: Sobel Operator gradient value

$G_{X}:$ Sobel Horizontal Gradient

$G_{y}:$ Sobel Vertical Gradient

The Prewitt operator is mostly similar with sobel operator used for detection of image edges in horizontal and vertical diretction.Prewitt operator also uses $3 \times 3$ uses matrixes in the direction of convolution masks considered to be in orthogonal direction. The Prewitt edge detection is a discrete differentiation operator that computes an approximate solution of the gradient of the input image. The Prewitt operator is based on transforming the data in the horizontal and vertical direction using a reduced distinctive and integer weighted filter and is there for extremely useful in the analysis of computations especially in comparison to the Sobel and kayyali operators.

$$
h_{1}=\left[\begin{array}{ccc}
1 & 1 & 1 \\
0 & 0 & 0 \\
-1 & -1 & -1
\end{array}\right] \quad h_{1}=\left[\begin{array}{lll}
-1 & 0 & 1 \\
-1 & 0 & 1 \\
-1 & 0 & 1
\end{array}\right]
$$

Usually, Sobel and Prewitt's methods are used to detect objects, but they are nor recommended owing to their noise sensitivity, which resulting in compromised accuracy. On the contrary, canny edge detection performs well in edge detection but with more computational requirements. The Canny edge detection provides better edge detection, better localization and direct response [11]. The Canny edge detection provides better edge detection, better localization and direct response [7]. In this paper, canny algorithm is selected for vehicle edge detection.

\section{B. Classification Approaches}

In geometry-based approach, vehicles are classified on the basis of its length, width, and height of its blobs. In this approach, vehicles are recognized as either car or non-car based on their length and height [9]. In mixed approaches vehicles are classified in two levels k-nearest neighbor classifier $(\mathrm{KNN})$ and adaptive neural network (NN). In order to differentiate the vehicle categories, the geometrical features such rectangularity such as aspect ratio, width, and area are used. During the initial stage, the size is calculated using KNN followed by vehicle type recognition. In addition, an adaptive $\mathrm{NN}$ is used to distinguish between small and big vehicle on the basis of the collected image information

The feature vector is a pattern recognition technique that is used to categorize vehicles into different classes. Feature extraction is helpful to extract out the features of an image the feature extraction has been applied to isolate the various Vehicles objects. Feature extraction, binary classification technique Binarization process calculated the threshold value to differentiate the object of interest from its background [12.15]. A feature vector is used to calculate area, length, and height, and centroid regions i.e., major axis minor axis. By using Region props thresholding vehicles are classified according to their type.

\section{System Design}

Given the research literature, this paper considers the following innovative vehicle classification system for its implementation on small computing devices with low power in consideration of future smart applications (using small Cyber Physical Devices where necessary). The overall workflow of the proposed system is displayed in Figure 1. The proposed approach is an alternative to traditional FPGA design flow using Xilinx system Generator which is difficult, time consuming and error prone. The proposed system-level model-based design shortens the development, debugging, and validation time. The proposed research methodology includes designing of a vehicle detection and classification model using Zedboard environment in MATLAB/Simulink. After that Simulink model of vehicle detection and classification algorithm is prepared and configured to be used for code generation. Once the configuration and parameters setting of the model is accomplished, VHDL code of design is generated using Xilinx system generator token Workflow Advisor. The VHDL code is synthesized for hardware realization and implemented on the FPGA 
Zedboard device.

- The input is taken from the "image file" MATLAB Computer vision block. Essentially, image pre-processing entails converting frames to serial.

- The frames are processed using an RGB to a grey algorithm based on a Xilinx model. Gaussian filter is applied on gray images to filter out unwanted noise from the image.

- Hysteresis thresholding is used to make the edge thick.

- A feature vector is used for the extraction of the feature of vehicles and an SVM classifier is used to classify vehicle type.

- $\quad$ Post-processing is done by Converting serial to frames in an image.

- The overall, model is developed into a system generator by using a system generator token to generate automatic VHDL code of that model.

- For further processing that code RTL view of that design can be viewed, design utilization summary and timing performance can be seen.
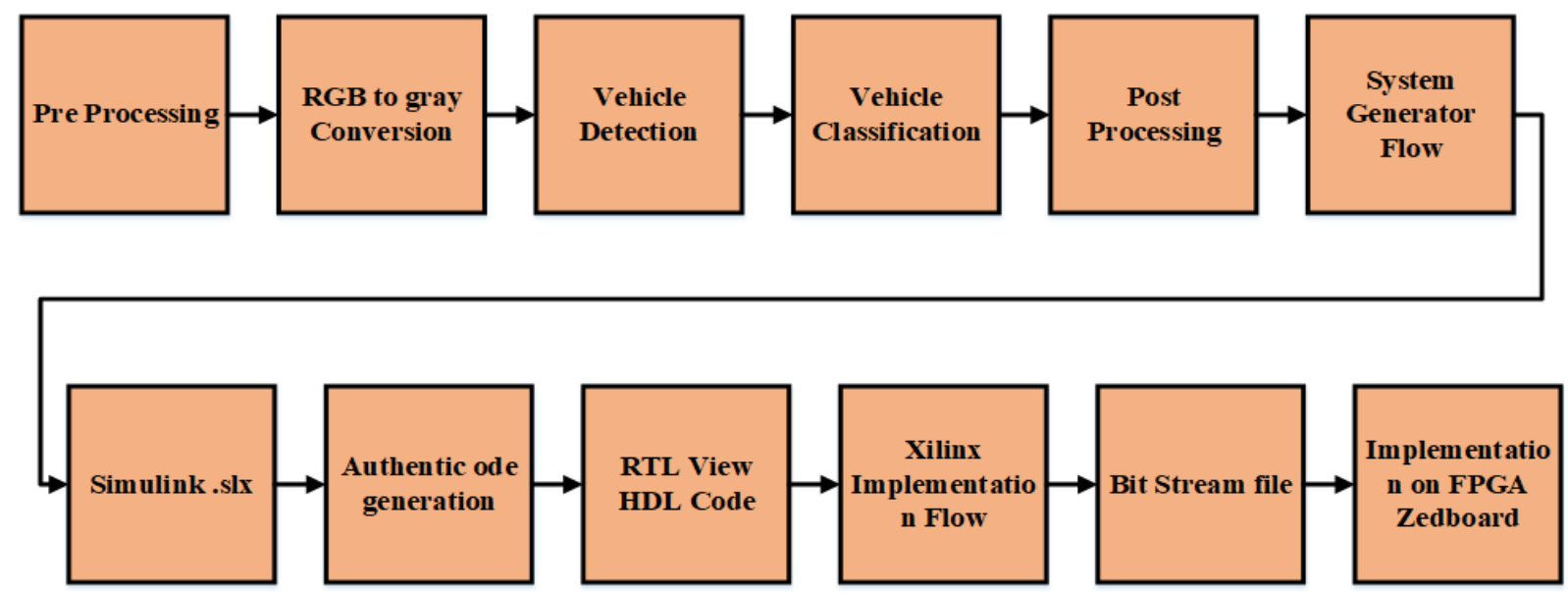

Fig. 1. Fpga Based Vehicle Detection and classsficationn model Block Diagram

\section{System Design Implementation}

The overall Simulink model of the system as shown in Fig. 2 contains a hardware co-simulation model of vehicle detection and classification for modules is discussed in subsequent sections. The overall Simulink model of the system as shown in Fig. 2 contains vehicle detection and classification modules are discussed in subsequent sections.

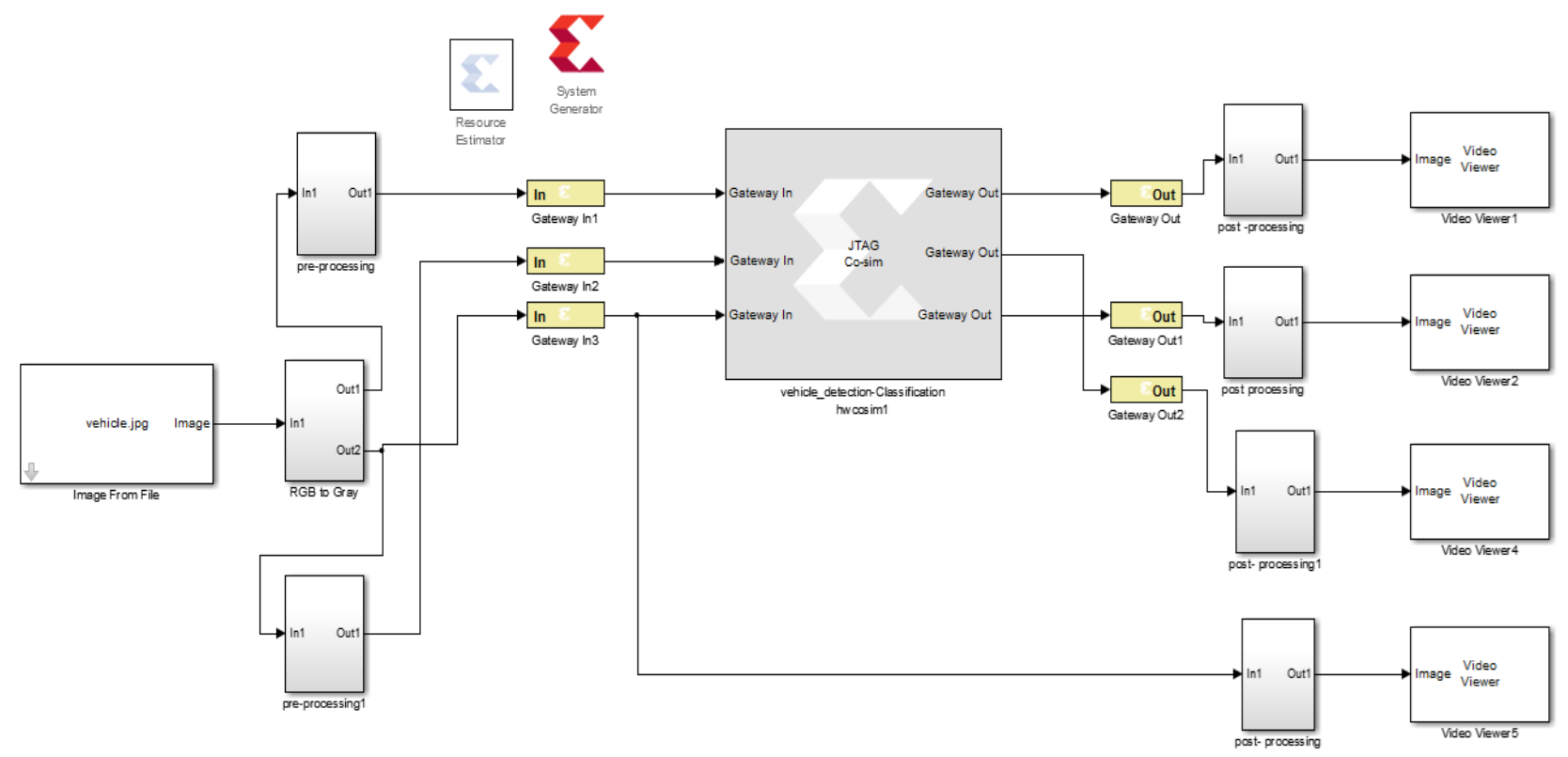

Fig. 2. Hardware -Co simulation model for vehicle detection and classification system using FPGA Zed board 
Proposed architecture is developed using Xilinx system generator blocks .Software used in the research are Matlab 2018 and vivado 18.2. Using hardware co -simulation tool.

RGB s image frame is given as an input to using image from file Xilinx Block. Pre-processing is done to convert original frames into serial data .Next step is RGB to Gray conversion using RGB intensity block .Gaussian filter is applied to the gray scale image frames to blurs the frames and noise effects Due to smoothing effects, gradient output contains lot of thick edges and None maximum suppression is applied to make the edges thin The hysteresis thresholding is the final block of the canny algorithm .which is based on two threshold level, i-e high and low, to suppress noise effects. After vehicle detection, features are extracted using binary feature extraction technique by applying SVM (support vector machine) classifier. Model based design of the proposed system is converted into VHDL (Verilog Hardware Description language The .VHDL code is generated automatically using system generator token. Complete model is ported into into vivado software .Hardware co-simulation is performed on zed board 7020, it is evaluated that hardware co -simulation is an easy and very interesting way in the field of FPG to design complex designs efficiently without paying any prize when implemented on hardwire. Hardware co-simulation is generated by simulation does not effects overall performance of the system .Area performance for vehicle detection and classification are analyzed and RTL and RTL technology view is reported .Hardware co- simulation model contains lot of subsystems: Image pre-processing ,RGB to gray conversion, ,Guassain filter, gradient calculation and ,hysteresis thresholding vehicle classification using M.code (Matlab Function block) and post processing .Each subsystem is described below in detail.

\section{A. Image from file}

Image pre-processing in Matlab used for providing input to FPGA. Image pre-processing in Matlab helps in providing input to FPGA. Image pre-processing is used to convert Image frames into Serial data. Image is read from the MATLAB environment using image from file block. Image pre-processing in Matlab helps in providing input to FPGA. 0 shows detailed steps for pre-processing.

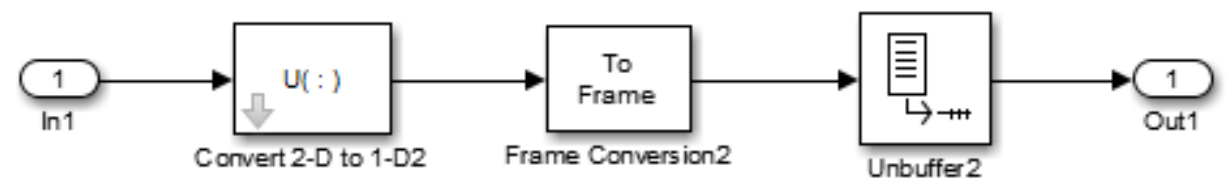

Fig.3. Image pre-processing

\section{B. RGB to Gray Conversion}

In image processing, the input image includes color or other unnecessary information which are considered as noise in edge detection. The second step is to reduce these noises by removing unwanted information. Although RGB to Gray conversion can reduce data but the gray scale image can still contain noise which further requires Gaussian filter.

\section{Gaussian filter}

Gaussian filter operation has been applied on RGB images The proposed design uses $5 \times 5$ the Gaussian filter is used for smoothing the noisy gray level image .

Gaussian filter acts a low pas filter used to eliminate noise and blocks high frequency components. Fig. 4 gives the design of the block.

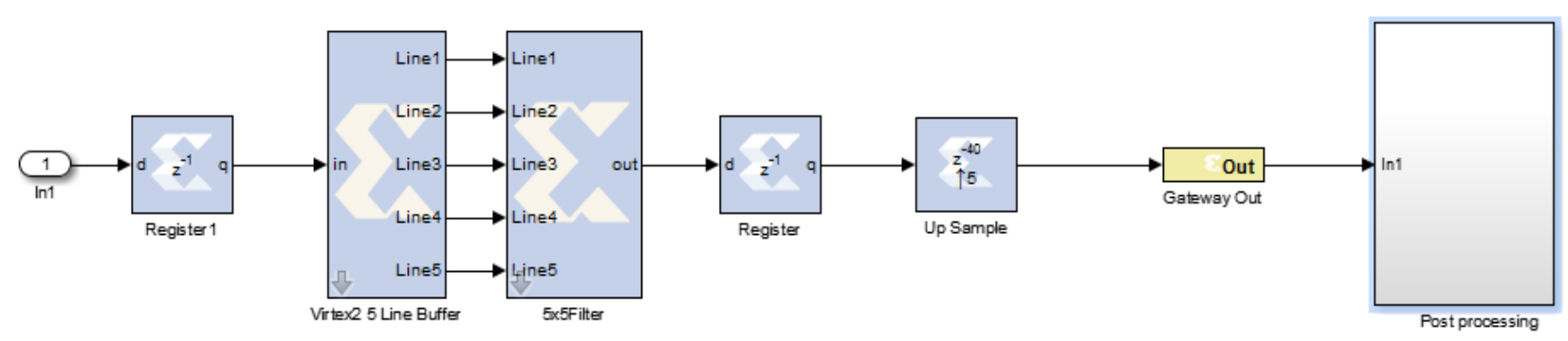

Fig.4. Gaussian Filter

\section{Hysteresis Thresholding}

Some edges caused by noises known as 'streaking' can be eliminated by hysteresis thresholding as shown in Fig. 5. 


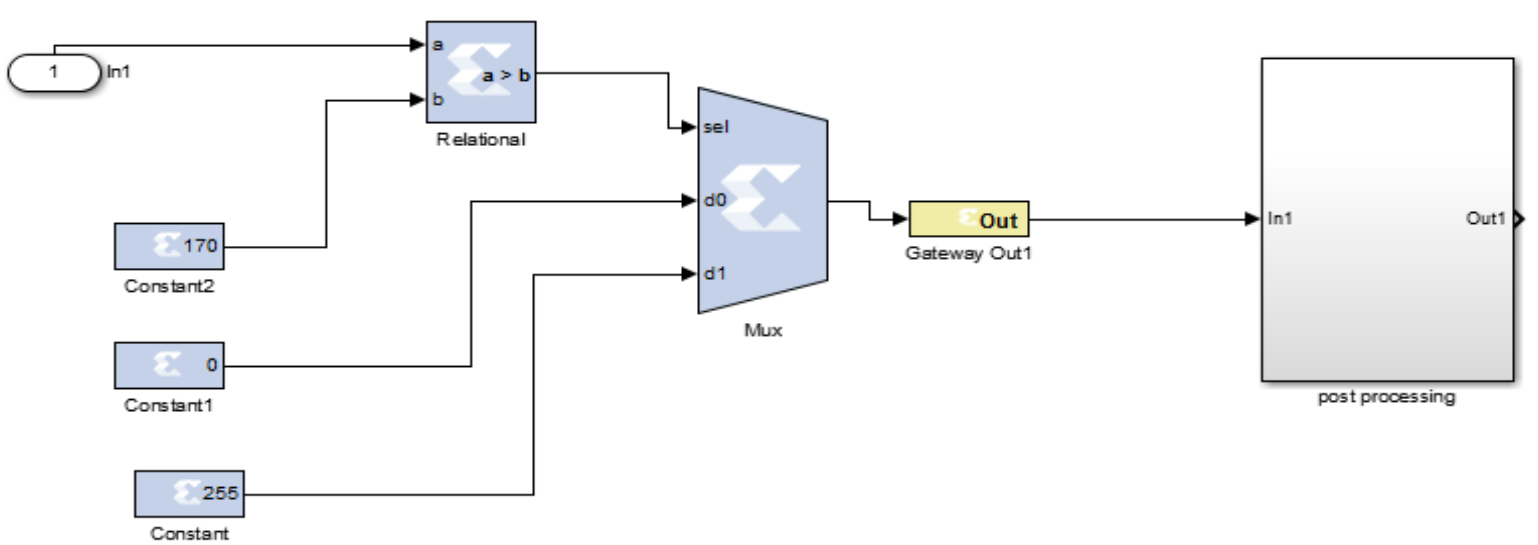

Fig.5. Hysteresis Thresholding

\section{E. Feature Vector Binary Thresholding}

For vehicle classification, the Feature vector technique is used [13]. Feature vector-based model is designed to calculate the number of objects in the image. By using function region props computes every object properties i.e., length, width, height, bounding box, centroid, filled area. Based on those properties, it computes some values. For vehicles based on region property value classify that vehicle using binary values as car, bus, etc. Matlab function block is used in system generator to import MATLAB code into Simulink model using the MATLAB Function Block. Matlab code is executed within the model to read the input data and then pass this data into Simulink block. Fig. 6 shows the feature vector binary thresholding $\mathrm{m}$ block.

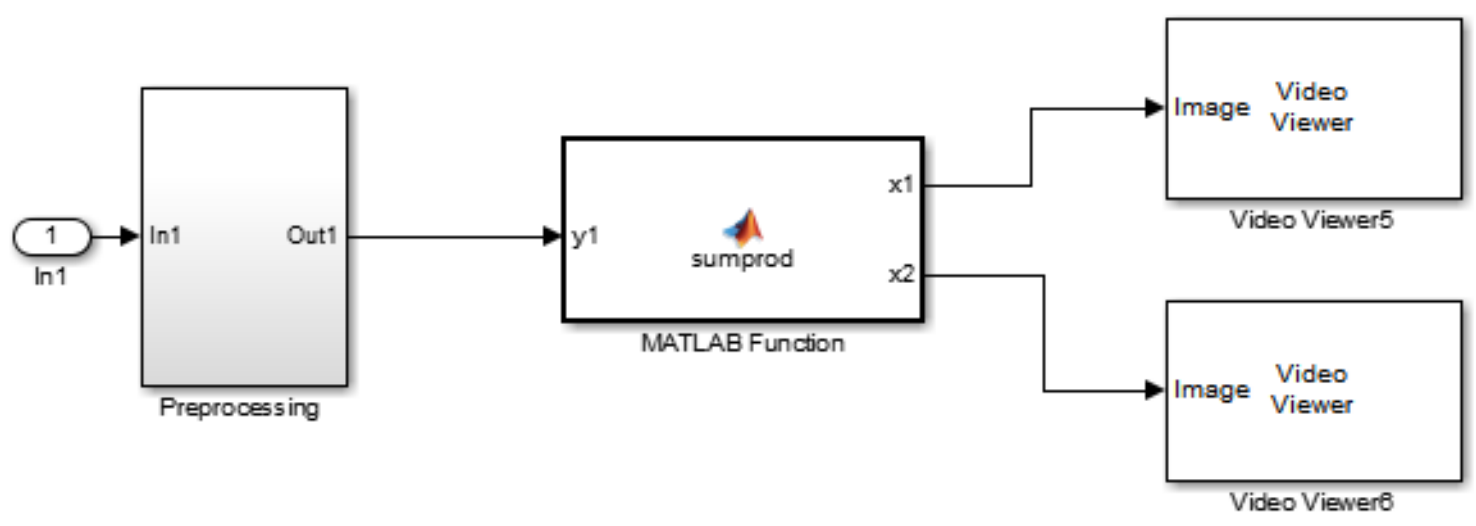

Fig.6. Feature Vector Binary Thresholding

\section{F. Post Processing}

The Image post processing block Fig. 7 is used for converting back serial data into frames for post processing detailed steps are shown below.

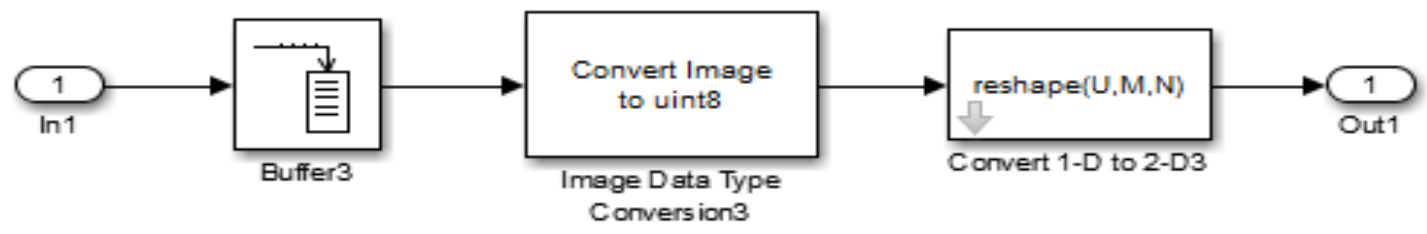

Fig.7. Post Processing 


\section{Results and Discussions}

The Simulink model of vehicle detection and classification is simulated in Zedboard Xilinx system Generator 14.7 and Matlab 2013a Environment. The work aims to detect and classify vehicles using an efficient algorithm. Extensive simulation were conducted and consistently expected results are obtained. Fig. 10 shows the original color image, which is chosen as test frame, its greyscale conversion of that image is shown in Fig. 8(c) and Fig. 8(d) shows the output image after applying the low pass filter that is Gaussian filter to remove noise and hysteresis thresholding, feature extraction, and finally fig show classification of the vehicle using SVM classifier.

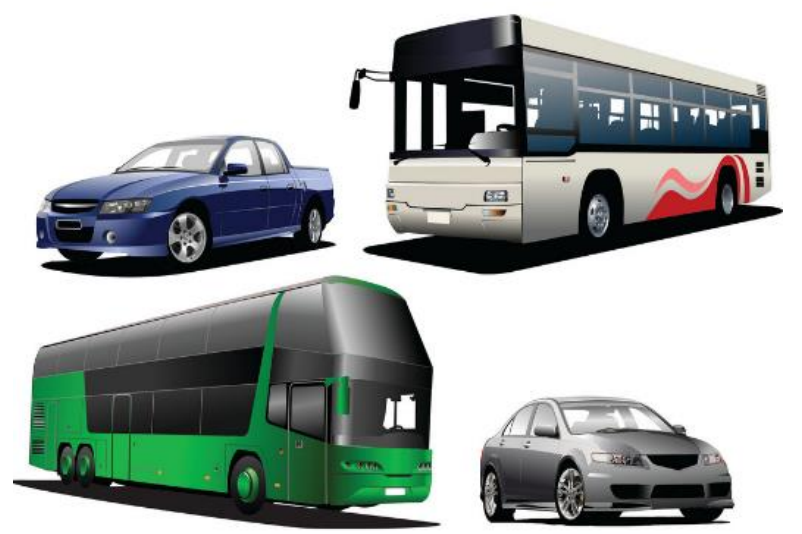

Fig.8. Vehicles Original color image

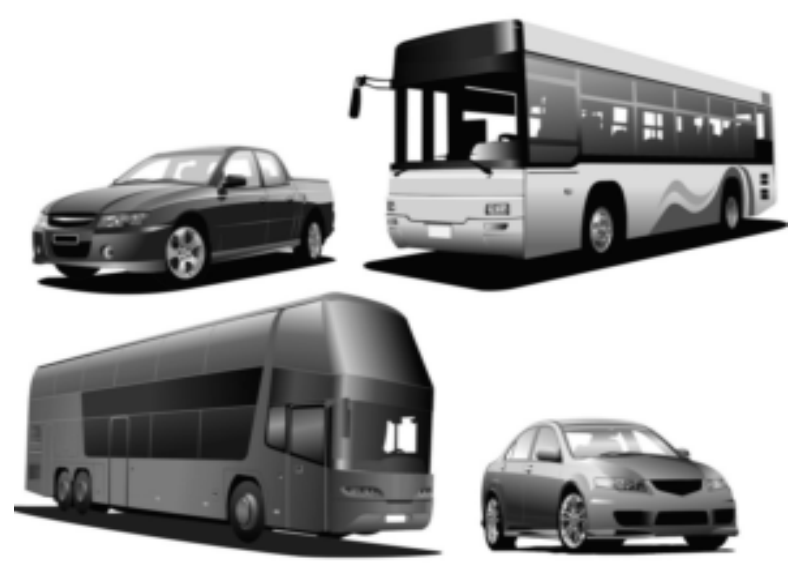

Fig.9. Vehicles Gray Scale Frame

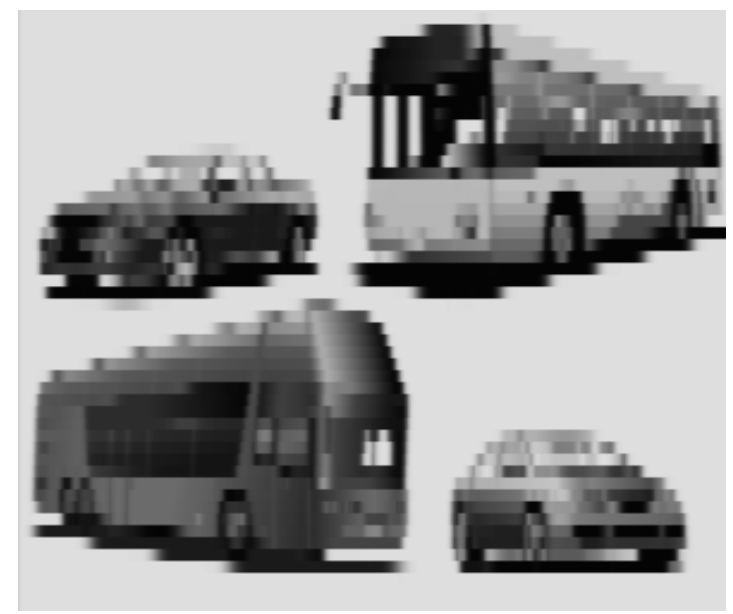

Fig.10. Gaussian filter based Vehicles Blurred Frame 


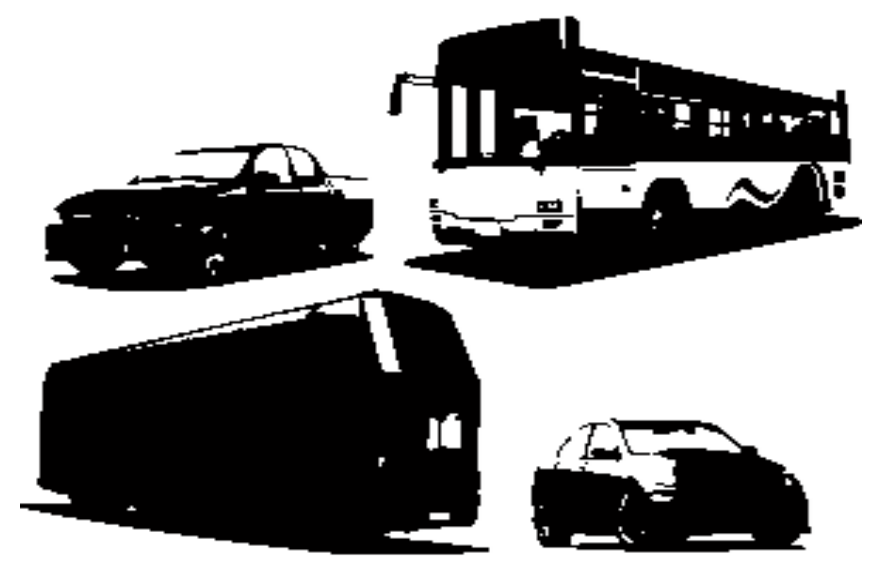

Fig.11. Vehicle Detection Frame

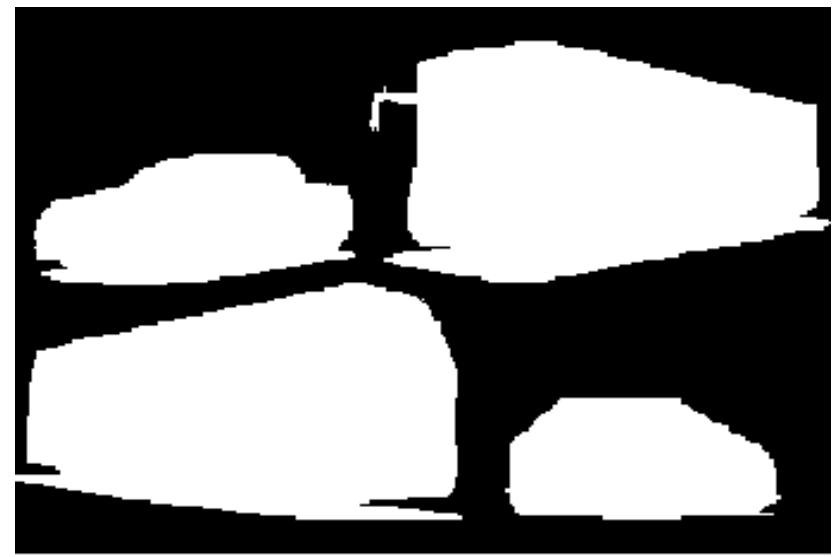

Fig.12 vehicle Feature extraction Frame

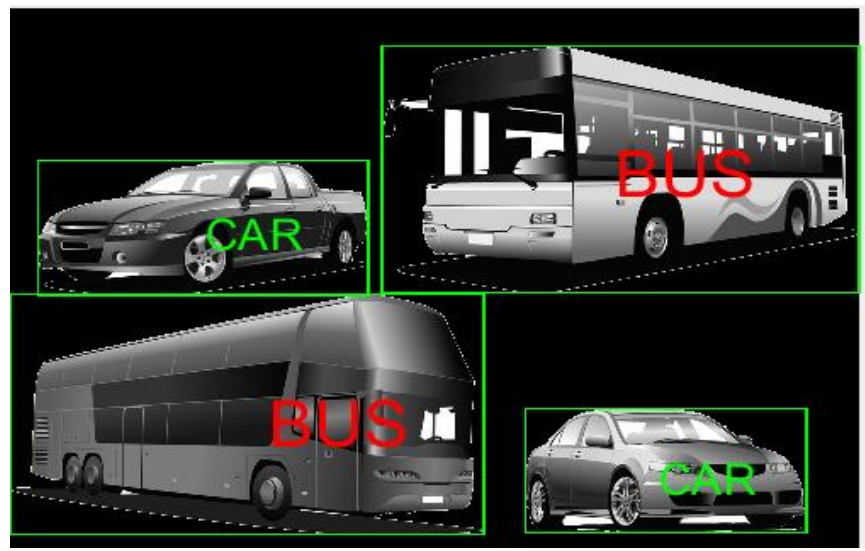

Fig.13.Vechile Classification

The proposed design of Vehicle detection and classification is implemented on Zedboard FPGA target device using Xilinx ISE Design Suite 14.7. The whole model of XSG block set was ported and tested on the Zed Board 7020 board. The VHDL code that was imported into Vivado 2016.4 software and netlist has been generated automatically.

The design utilization summary of the proposed Vehicle detection and classification is given in Table 1. From the table it is observed that proposed design occupies 64 slice registers, 1167 look up tables and 100 bonded input outputs of the device. Hence a small chunk of FPGA Resources (i.e. < 5\%) is used by the model. As the hardware consumption determines the cost of any design, the proposed design utilizes less hardware and thus it is cost effective. Table- 2 shows the timing performance of the design. The hardware implementation of the vehicle detection and classification has clock speed of $128 \mathrm{MHZ}$ with the minimum period of $8 \mathrm{~ns}$. It can also be assessed that hardware co-simulation is an easy and interesting way of designing complex designs that do not pay the price for efficiency when implemented on hardware. 
The code that the system generator generates does not reduce overall performance. RTl technology view is shown in Fig. 10. The technology view of the proposed design generated in the FPGA is shown in Fig. 11.

Table 1. Fpga based vehicle detection and classification Area Utilization

\begin{tabular}{|l|l|l|l|}
\hline Slice Logic & Available & Used & Utilization \\
\hline Number of slice registers & 35,200 & 717 & $2 \%$ \\
\hline Number of slice LUTS & 17,600 & 517 & $2 \%$ \\
\hline Number of slice LUT-FF pairs & 712 & - & - \\
\hline Number of bounded IOB'S & 33 & 100 & $30 \%$ \\
\hline Number of block Ram/ffo & 60 & - & - \\
\hline $\begin{array}{l}\text { Number of } \\
\text { BUFG/BUFGCTRLS }\end{array}$ & 32 & 1 & $3 \%$ \\
\hline Number of occupied slices & 215 & 4,400 & $4 \%$ \\
\hline
\end{tabular}

Table 2.Performance Utilization

\begin{tabular}{|l|l|}
\hline Parameter & Value \\
\hline Minimum Period & $8 \mathrm{~ns}$ \\
\hline Maximum Frequency & $128 \mathrm{MHZ}$ \\
\hline
\end{tabular}

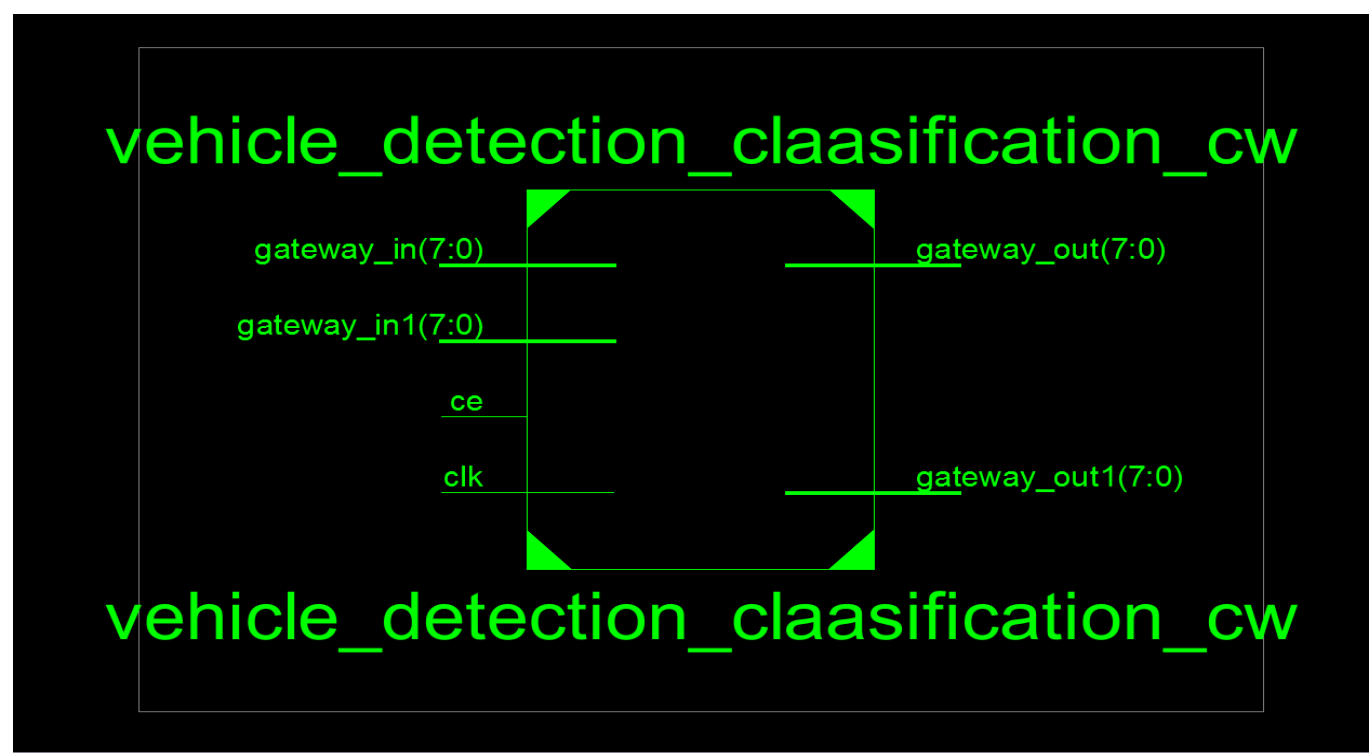

Fig.14. Design Abstraction RTL view

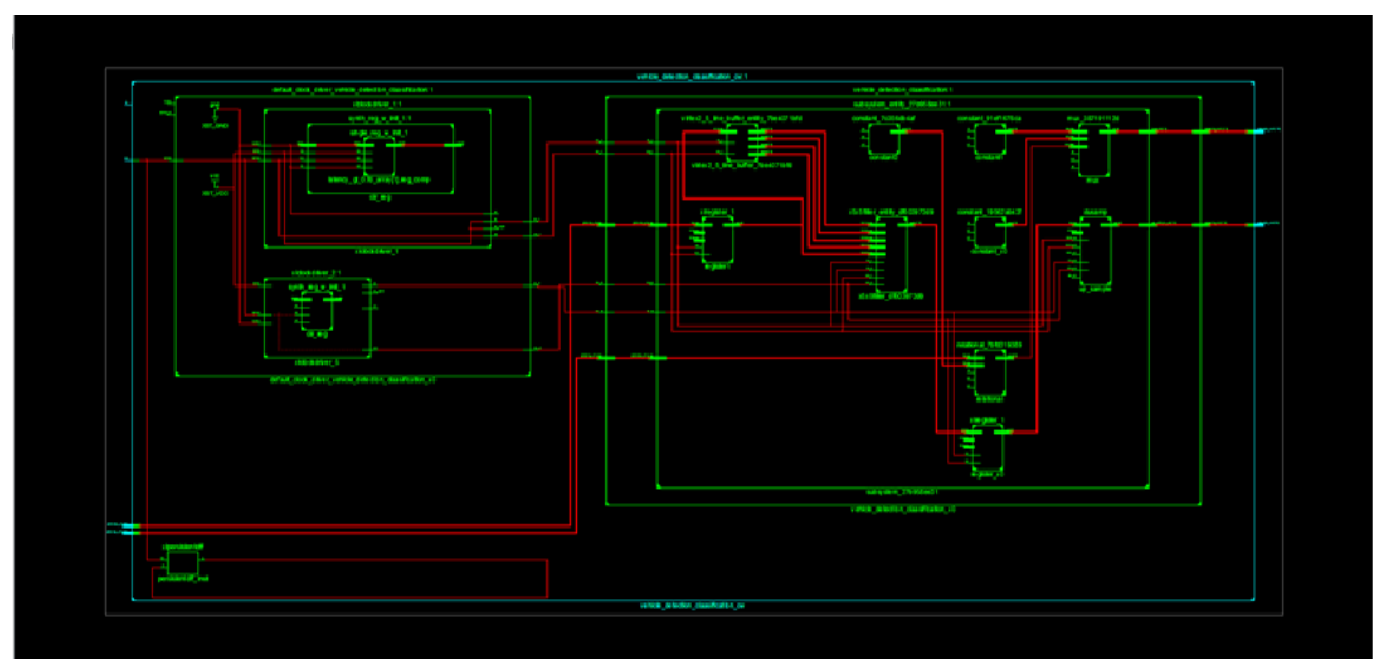

Fig.15.Design Abstraction RTL Technology View 


\section{Conclusion}

In this work, Vehicle detection and classification system is developed in MATLAB/Simulink using canny edge detecting algorithm and feature vector classification. Proposed design is then successfully implemented on Xilinx Zedboard Zynq xc7z010-1clg400 FPGA using the Xilinx System Generator. This design technique not only improves the product development cycle, but also provides a selection of design optimization alternative solution and trade off allowing for achievement of predefined performance criteria when compared to manual coding, which is difficult and time consuming. The design methodology is efficient and provides a high level of performance. It is observed that canny edge detection technique provides sharp edge detection. The implemented design on Zedboard FPGA can perform at an estimated frequency of $128 \mathrm{MHZ}$ and consume less power. For hardware resources, Area performance Utilization and RTL design of proposed system design is reported successfully. Design optimization is also performed in terms of speed, area, and power. Through this FPGA platform we have achieved high performance, lower cost and the efficient system compared to contemporary counterpart. Point to the future is for real time implementation and its functional verification using standalone embedded system. Future work extended for the implementation of vehicle detection and classification on a larger scale.

\section{References}

[1] S. Kul, S. Eken, and A. Sayar, "A concise review on vehicle detection and classification," Proc. 2017 Int. Conf. Eng. Technol. ICET 2017, vol. 2018-January, no. January 2019, pp. 1-4, 2018, doi: 10.1109/ICEngTechnol.2017.8308199.

[2] M. P. H. Pawar and P. R. P. Patil, "FPGA Implementation of Canny Edge Detection Algorithm," Int. J. Eng. Comput. Sci., vol. 3, no. 10, pp. 8704-8709, 2014.

[3] G. N. Chaple, R. D. Daruwala, and M. S. Gofane, "Comparisions of Robert, Prewitt, Sobel operator based edge detection methods for real time uses on FPGA," Proc. - Int. Conf. Technol. Sustain. Dev. ICTSD 2015, no. 1, pp. 31-34, 2015, doi: 10.1109/ICTSD.2015.7095920.

[4] S. M. Alex Raj and M. H. Supriya, "Hardware Co-simulation of underwater moving object detection using Xilinx system generator," Int. J. Ocean. Oceanogr., vol. 10, no. 1, pp. 73-80, 2016.

[5] S. G. Kavitkar, "FPGA based Image Feature Extraction Using Xilinx System Generator," Int. J. Comput. Sci. Inf. Technol., vol. 5, no. 3, pp. 3743-3747, 2014.

[6] T. Kryjak, M. Komorkiewicz, and M. Gorgon, "Real-time hardware-software embedded vision system for ITS smart camera implemented in Zynq SoC," J. Real-Time Image Process., vol. 15, no. 1, pp. 123-159, 2018, doi: 10.1007/s11554-016-0588-9.

[7] S. Yaman, M. Yildirim, B. Kamişlioğlu, Y. Erol, and H. Kürüm, "Image and video processing applications using Xilinx system generator," 7th Int. Symp. Digit. Forensics Secur. ISDFS 2019, 2019, doi: 10.1109/ISDFS.2019.8757540.

[8] G. Baraskar and P. Thakre, "Evaluation of Canny and Sobel Edge Detection Technique using Xilinx System Generator," vol. 2, no. 2, pp. 53-56, 2017, [Online]. Available: http://ijsrst.com/paper/872.pdf.

[9] A. Arinaldi, J. A. Pradana, and A. A. Gurusinga, "Detection and classification of vehicles for traffic video analytics," Procedia Comput. Sci., vol. 144, pp. 259-268, 2018, doi: 10.1016/j.procs.2018.10.527.

[10] V. Keerthi Kiran, P. Parida, and S. Dash, Vehicle detection and classification: A review, vol. 1180 AISC, no. January. Springer International Publishing, 2021.

[11] L. Yuan and X. Xu, “Adaptive Image Edge Detection Algorithm Based on Canny Operator," Proc. - 2015 4th Int. Conf. Adv. Inf. Technol. Sens. Appl. AITS 2015, pp. 28-31, 2016, doi: 10.1109/AITS.2015.14.

[12] P. P. V. Keerthi Kiran, "Vehicle Detection and Classification: A Review"."

[13] S. Thepade, R. Das, and S. Ghosh, "A Novel Feature Extraction Technique Using Binarization of Bit Planes for Content Based Image Classification,” J. Eng. (United Kingdom), vol. 2014, 2014, doi: 10.1155/2014/439218.

[14] G. Baraskar and P. Thakre, "Evaluation of Canny and Sobel Edge Detection Technique using Xilinx System Generator," vol. 2, no. 2, pp. 53-56, 2017, [Online]. Available: http://ijsrst.com/paper/872.pdf.

[15] K. Kumar, R. K. Mishra, and D. Nandan, "Efficient Hardware of RGB to Gray Conversion Realized on FPGA and ASIC," Procedia Comput. Sci., vol. 171, no. 2019, pp. 2008-2015, 2020, doi: 10.1016/j.procs.2020.04.215.

[16] A. R. Bhagat, S. R. Dixit, M. T. Student, T. Engineering, and T. Engineering, "VHDL based Sobel Edge Detection," vol. 3, no. 1, pp. 1217-1223, 2015.

[17] Q. Xu, S. Varadarajan, C. Chakrabarti, and L. J. Karam, "A distributed canny edge detector: Algorithm and FPGA implementation,” IEEE Trans. Image Process., vol. 23, no. 7, pp. 2944-2960, 2014, doi: 10.1109/TIP.2014.2311656.

[18] K. Bala Krishnan, S. Prakash Ranga, and N. Guptha, "A Survey on Different Edge Detection Techniques for Image Segmentation," Indian J. Sci. Technol., vol. 10, no. 4, 2017, doi: 10.17485/ijst/2017/v10i4/108963.

[19] R. Mehra and R. Verma, "Area Efficient FPGA Implementation of Sobel Edge Detector for Image Processing Applications," Int. J. Comput. Appl., vol. 56, no. 16, pp. 7-11, 2012, doi: 10.5120/8973-3086.

[20] S. Parveen and S. Lasrado, "Computation of traffic density in video streams using XSG blockset," RTEICT 2017 - 2nd IEEE Int. Conf. Recent Trends Electron. Inf. Commun. Technol. Proc., vol. 2018-January, pp. 1248-1252, 2017, doi: 10.1109/RTEICT.2017.8256798.

[21] H. M. Abdelgawad, M. Safar, and A. M. Wahba, "High level synthesis of canny edge detection algorithm on Zynq platform," Int. J. Comput. Electr. Autom. Control Inf. Eng., vol. 9, no. 1, pp. 148-152, 2015, [Online]. Available: http://www.waset.org/publications/10000239. 
[22] S. Vijayarani, M. M. Vinupriya, and A. Professor, "Performance Analysis of Canny and Sobel Edge Detection Algorithms in Image Mining," Int. J. Innov. Res. Comput. Commun. Eng. (An ISO Certif. Organ., vol. 3297, no. 8, pp. 1760-1767, 2007, [Online]. Available: www.ijircce.com.

[23] S. Karanwal, "Implementation of Edge Detection at Multiple Scales," Int. J. Eng. Manuf., vol. 11, no. 1, pp. 1-10, 2021, doi: 10.5815/ijem.2021.01.01.

[24] R. R, N. Saklani, and V. Verma, "A Review on Edge detection Technique 'Canny Edge Detection," Int. J. Comput. Appl., vol. 178, no. 10, pp. 28-30, 2019, doi: 10.5120/ijca2019918828.

[25] R. Javadzadeh, E. Banihashemi, and J. Hamidzadeh, "Fast Vehicle Detection and Counting Using Background Subtraction Technique and Prewitt Edge Detection," Int. J. Comput. Sci. Telecommun. J. Homepage www.ijcst.org, vol. 6, no. 10, pp. 8-12, 2015, [Online]. Available: http://www.ijcst.org/Volume6/Issue10/p2_6_10.pdf.

[26] D. Kumari and K. Kaur, "A Survey on Stereo Matching Techniques for 3D Vision in Image Processing," Int. J. Eng. Manuf., vol. 6, no. 4, pp. 40-49, 2016, doi: 10.5815/ijem.2016.04.05.

[27] N. Kumala Dewi, "Review of Vehicle Surveillance Using Iot in the Smart Transportation Concept," Int. J. Eng. Manuf., vol. 11, no. 1, pp. 29-36, 2021, doi: 10.5815/ijem.2021.01.04.

\section{Authors' Profiles}

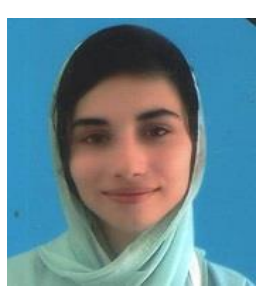

Aisha Baloch is Electronics engineer. Currently, she is doing Master of Engineering in Electronic Systems Engineering and received the B.E degree in Electronic Engineering from Mehran University of engineering and technology Jamshoro. Author has research interest in smart cities, FPGA based system design, Image Processing, Artificial Intelligence, Embedded Systems, Internet of things, Machine Learning and Deep Learning.

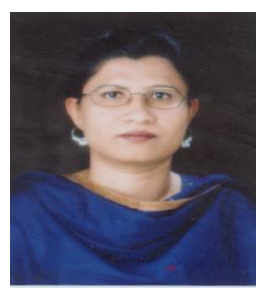

Dr. Farida Memon is an Associate Professor in Department of Electronics Engineering Mehran University of Engineering \& Technology Jamshoro, Sindh Pakistan, having more than 25 years of teaching \& research experience. She holds a PhD in Electronic Engineering from Mehran University of Engineering \& Technology (MUET) Jamshoro, Pakistan. She Received the B.E degree in Electronic Engineering and M.E degree in Telecommunication and Control 1993 and 2008 respectively. She has 33 National \& International Journal and Conference publications. Her research interest lies in FPGAs, Image Processing, Machine Learning, Deep Learning, Embedded and Digital Systems Design.

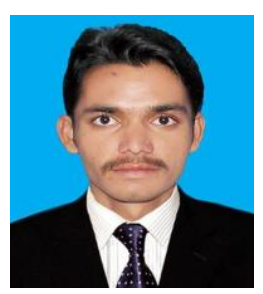

Ved Viyas is and Electronics engineer He Received the B.E degree in Electronic Engineering from Mehran University of engineering and technology Jamshoro. Author has research interest in smart cities, FPGAs, Image Processing, Automation and control Artificial Intelligence and Embedded Systems, Internet of Things and Machine Learning.

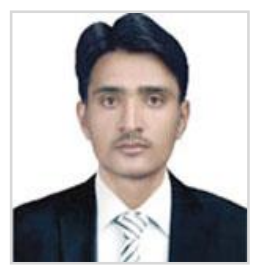

Bharat Lal is Lecturer at Electronic Engineering department, Mehran University of Engineering and Technology, Pakistan. Author has research interest in smart cities, Automation and control, recycling of waste material, Machine Learning, Artificial Intelligence and Embedded Systems

How to cite this paper: Aisha Baloch, Tayab D Memon, Farida Memon, Bharat Lal, Ved Viyas, Tony Jan, " Hardware Synthesize and Performance Analysis of Intelligent Transportation Using Canny Edge Detection Algorithm", International Journal of Engineering and Manufacturing (IJEM), Vol.11, No.4, pp. 22-32, 2021. DOI: 10.5815/ijem.2021.04.03 